\title{
Corporate profile: RoosterBio, Inc.
}

\author{
Timothy R Olsen*,1 \& Jon A Rowley ${ }^{1}$ \\ ${ }^{1}$ RoosterBio, Inc. 5295 Westview Drive, Suite 275, Frederick, MD 21703, USA \\ *Author for correspondence: tim@roosterbio.com
}

RoosterBio, Inc. (MD, USA) is a privately held stem cell tools and technology company focused on accelerating the development of a sustainable regenerative medicine industry, one customer at a time. RoosterBio's products are high-volume and well-characterized adult human mesenchymal stem/stromal cells (hMSCs) paired with highly engineered media systems. RoosterBio has aimed to simplify and standardize how stem cells are purchased, expanded and used in the development of regenerative medicine products. To this end, RoosterBio supplies off-the-shelf cGMP hMSC working cell banks with bioprocess media that mimic the format and formulation of the research grade counterparts, radically simplifying and shortening product development and clinical translation. RoosterBio's focus is to offer innovative products that help usher in a new era of productivity and standardization into the field, with a passion directed towards empowering life-saving cures to be discovered in regenerative medicine.

First draft submitted: 18 July 2018; Accepted for publication: 23 August 2018; Published online: 5 October 2018

Keywords: cell therapy • mesenchymal stem cells • regenerative medicine • stem cell

RoosterBio, Inc. (MD, USA) was founded in 2013 and focuses on human mesenchymal stem/stromal cell (hMSC) technologies, scalable biomanufacturing and streamlining clinical translation. RoosterBio is fueled by a vision of a future where living cell-based regenerative medicines are a major component of healthcare, both for treating diseases, and for preventing them. To realize this vision, these 'living cellular medicines' will need to be manufactured at commercially relevant scales using robust processes, and with a cost structure that supports reimbursement in an increasingly cost-sensitive global healthcare landscape. To address these challenges, RoosterBio has commercialized supply chain innovations that radically reduce the development timelines of living cellular medicines, addressing the most significant industry bottleneck related to cell supply and scalable manufacturing, thus enabling product developers to routinely generate billions of therapeutically relevant cells for development, clinical application and, ultimately, commercialization. Simply put, RoosterBio's goal has been to hypersimplify the incorporation of living cells into medical products by reducing process and product development timelines for hMSC-based therapeutics.

In late 2013, RoosterBio launched operations inside the Frederick Innovative Technology Center, Inc., and shipped its first products to customers in early 2014. These early 'first-in-class' products comprised large volumes of working cell banks and paired bioprocess media systems engineered for the rapid and robust manufacturing of billions of high quality hMSCs, finally eliminating the critical bottleneck of cell generation that had been inhibiting product development for decades. RoosterBio's paired cell and bioprocess media systems triple to quadruple cell productivity and drastically reduce the cost structure for hMSC manufacturing.

Starting with half a dozen employees, RoosterBio grew to 18 employees prior to graduating from the Frederick Innovative Technology Center, Inc., technology incubator in early 2018 and moving into a custom-built 14,000 square foot facility designed to accommodate the highest level of innovation in stem cell manufacturing, research and development, and process development. As of June 2018, RoosterBio has 28 full-time employees, with 12 $\mathrm{PhDs}$ on staff. The team has extensive expertise in all aspects of tissue engineering, regenerative medicine and cell therapy manufacturing and are viewed as leaders in the field [1-5]. As such, RoosterBio has filed several patent applications related to cell manufacturing unit operations [6-8].

Future Medicine 


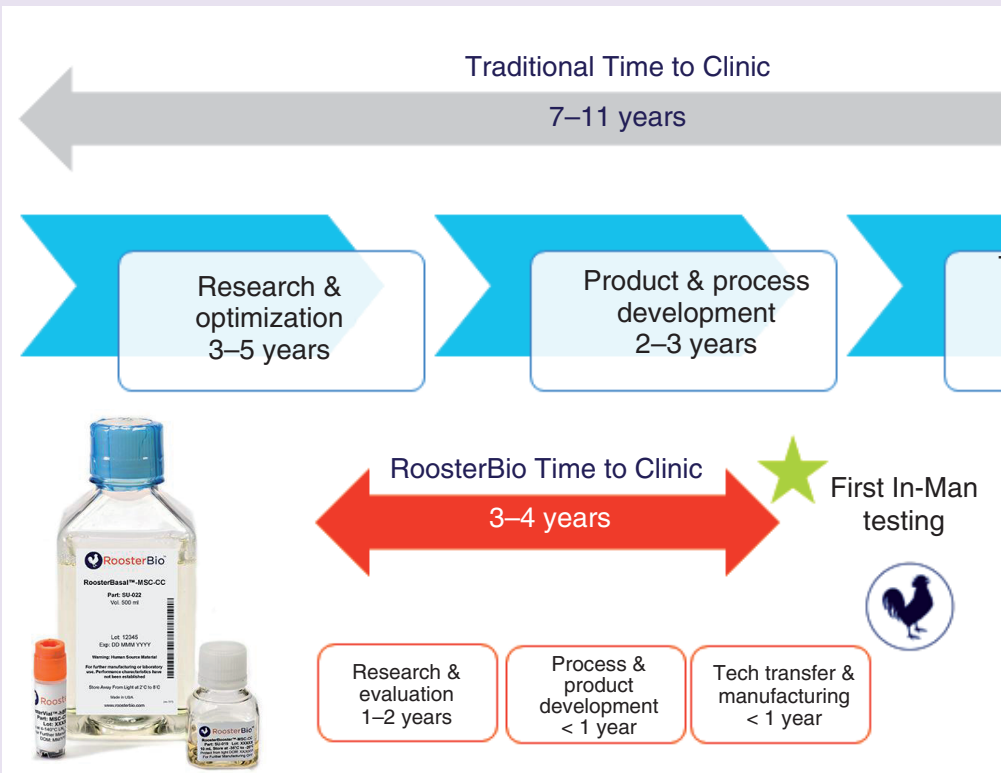

First In-Man Testing

Figure 1. RoosterBio aims to radically streamline the current typical timeline for research, development and clinical translation.

\section{Strategy: RoosterBio is 'microchipping mesenchymal stem/stromal cells'}

To grasp the potential of hMSCs to drive the regenerative medicine industry, it can be helpful to consider them in terms of another ubiquitous technology: microchips. Like microchips, RoosterBio views hMSCs not as tools for research, but as complicated pieces of technology. The hMSCs necessitate controlled and scalable manufacturing processes with defined parameters that lend to their critical quality attributes. To date, hMSCs have been used to treat over 36,000 patients globally [9] and there are currently over 900 registered global clinical trials utilizing hMSCs [9], with 100 new trials added each year since 2011 [10]. The hMSCs are being tested for a wide variety of therapeutic applications, from bone and cartilage regeneration, to treating stroke, cardiovascular disease and to combatting cancer. Similar to how advancements in microchip manufacturing, coupled with increased ease of implementation, have enabled logarithmic growth in the computing industry over the last few decades, giving rise to new technologies and applications never before imagined, hMSCs are poised to drive explosive growth of the regenerative medicine industry and radically advance the standard of medical care.

\section{Accelerating development of the field}

RoosterBio's strategy is to accelerate the development of regenerative medicine by simplifying the incorporation of hMSCs into next generation regenerative medicine products. RoosterBio does this by providing large quantities of high quality human therapeutic cells in simple, abundant and affordable formats for key technology areas including tissue engineering, cell therapy, bioprinting, extracellular vesicle and synthetic biology applications. RoosterBio's platform technology is based on large volume allogenic working cell banks paired with highly efficient bioprocess media and standardized manufacturing processes. The technology has been simplified through product design with end-user applications and scalability in mind, effectively making any user an hMSC manufacturing expert overnight. A customer-centric technical support system is also in place with pro-bono manufacturing process development consulting available to customers. Providing large volume, high-quality starting material in scalable formats helps the end user to achieve higher cell numbers faster, transitioning hMSCs from a scarce resource into an abundant resource, reducing development timelines and, ultimately, contributes to the acceleration of the industry. This state of hMSC abundance should enable regenerative medicine technologies to get to market faster and more economically, while also allowing product developers to implement hMSCs in unprecedented novel applications. In addition, RoosterBio is paving the way for rapid clinical translation and commercialization by providing tools for readily translating research and development work from the bench to the clinic with its cGMP clinical-grade 
media and the only commercially available, off-the-shelf cGMP clinical-grade hMSCs. By eliminating technical barriers and providing standardized manufacturing solutions to remove the tremendous redundancy in the field related to hMSC products, RoosterBio hopes to have the same impact on the regenerative medicine industry that Intel had on the computing industry.

\section{Partnerships}

To increase its reach and impact, RoosterBio has strategically partnered with industry leaders in the cell manufacturing space to provide novel end-to-end solutions for its customers. In 2017, RoosterBio named GenCure, a San Antonio-based cell and tissue processing contract manufacturing organization with cGMP compliant manufacturing and processing capabilities, as its preferred manufacturer, providing customers access to cGMP manufacturing of their final product, leveraging RoosterBio's scalable technology platform, for rapid initiation of first-in-man studies. In addition, RoosterBio recognizes that 'next generation' hMSC products, already on the horizon, will harness the hMSC secretome and has therefore partnered with an exosome industry leader, ExoPharm, to initiate a Stem Cell Exomere program, with the goal to bring high-grade extracellular vesicles/exosomes derived from adult stem cells into clinical practice. Furthermore, an avant-garde partnership with CELLINK, the first company to commercially produce hydrogel bioink, has allowed RoosterBio to address critical bottlenecks for the bioprinting community, who require tremendous quantities of cellular bioink as they look toward tissue and whole organ manufacturing. Finally, partnering with Pall Corporation and PBS Biotech has enabled RoosterBio to advance and optimize bioreactor platform technologies for scalable manufacturing of clinically and commercially relevant lot sizes for adherent cells.

\section{National consortia}

In addition to industry partnerships, RoosterBio participates in several national consortia, including the National Institute for Innovation in Manufacturing Biopharmaceuticals, the National Institute of Standards and Technology, the Alliance for Regenerative Medicine, the National Science Foundation Engineering Research Center for Cell Manufacturing Technologies (CMaT), and the Advanced Regenerative Manufacturing Institute to drive innovation in manufacturing sciences, democratization and standardization of stem cell technologies and making practical the large scale manufacturing of engineered tissues, all in an effort to pave the road for the future of regenerative medicine as an industry. RoosterBio has secured significant nondilutive grant funding and government contracts through the NIH, the Maryland Stem Cell Research Fund and the Medical Technology Enterprise Consortium. This nondilutive funding validates the company mission, vision for the field and the technology approach RoosterBio is using to advance the regenerative medicine field.

\section{RoosterBio corporate capabilities \& key technologies}

RoosterBio's expertise is in stem cell tools, technologies and manufacturing processes. Combining this with decades of cumulative industry experience, RoosterBio is capable of developing clinically and commercially viable biomanufacturing processes, and hopes to contribute to the booming regenerative medicine industry in the years to come. RoosterBio continues to refine scalable processes for in-house hMSC manufacturing but also strives to assist customers through its development services arm and aids the development of custom large-scale manufacturing processes, subsequently bringing these processes back in-house via tech transfer from RoosterBio.

RoosterBio's product portfolio includes not only large volume hMSC banks, but also a number of other firstto-market product formats including: 'ready to print' cells (the first true 'thaw and use' stem cell reagent), donor screening kits, batch and fed-batch bioprocess media systems (including the industry's only stem cell bioreactor feed), bioreactor kits, bioprinting kits, preclinical development kits, and cGMP clinical-grade hMSCs and media. RoosterBio aims to ensure its products are ahead of their time and strives to repeatedly set the industry standard and provide much-needed solutions to product developers as and when they need them. A prime example is the aforementioned expanded, ready-to-print cell product, delivered at 50 million cells per vial and ready to use immediately upon thaw (for directly seeding onto constructs and medical devices or for use in 3D bioprinting applications). This product effectively removes the need for a cell culture infrastructure, significantly streamlining workflows for tissue engineers and bioprinters.

A key, and highly differentiated, product design requirement for any RoosterBio offering is that it must afford the customer a smooth transition to the clinic. From day 1, RoosterBio has focused on ensuring products are manufactured to the highest industry standards utilizing clinically relevant processes, reagents and materials, 
thereby minimizing the benchtop-to-bedside chasm that seems to have crippled the translation and maturation of cell-based therapeutics. To this end, RoosterBio's research-use-only xeno-free hMSCs and bioprocess media systems are analogous to its cGMP hMSCs and bioprocess media systems in terms of formulation, configuration and manufacturing process. This streamlines product development and clinical translation for the end user, enabling RoosterBio customers to progress to the clinic with their regenerative medicine products in a fraction of the time it would have otherwise taken (Figure 1). RoosterBio is further supporting customers' clinical translation with registered US FDA master files for its cells and media. These reference documents will streamline the end user CMC submission process by allowing the customer to simply reference these documents during an investigational new drug filing process. RoosterBio's cGMP hMSCs and bioprocess media are a major paradigm shift in the hMSC supply chain and should fuel rapid growth in hMSC clinical trial initiation and progression to commercialization.

\section{Future perspective}

The MSCs are poised to have tremendous impact on regenerative medicine applications ranging from tissue engineering to gene editing. There are over 900 registered clinical trials utilizing hMSCs [9], with more than 100 trials being added each year since 2011 [10], and an average of 416 million cells used per patient [11]. By enabling large-scale economic manufacturing of hMSCs, RoosterBio's goal is to drive a log increase (9000) in the number of hMSC clinical trials over the next 10 years. The momentum for hMSC-based technologies is growing, and manufacturing sciences are paving the way to improved economies of scale, reduced cost of goods and democratized hMSC-based products. Widespread abundance of a standardized hMSC supply chain will enable this technology to be readily incorporated into new applications and next generation hMSC products. Just as the computing revolution was ushered in by the availability and standardization of microchip technology, availability and standardization of hMSCs will enable them to become the microchips of tomorrow's regenerative medicine products. RoosterBio aims to be a leading provider of hMSC technologies to fuel this regenerative medicine revolution. RoosterBio will continue to develop and deliver products focused on simplifying product and process development, enabling scalable cell manufacturing, new application development, and shortening the time to the clinic and market.

\section{Summary points}

- RoosterBio, Inc. is a pioneer in regenerative medicine, with a focus on human mesenchymal stem cell (hMSC) raw materials, bioprocess design, and manufacturing technologies that was founded to address the industry wide bottle neck that is the scarcity of clinically relevant starting materials for product development.

- RoosterBio's strategy is to accelerate the development of regenerative medicine by simplifying the incorporation of hMSCs into next generation regenerative medicine products. RoosterBio does this by providing off-the-shelf working cell banks with a robust regulatory profile that are simple to implement into regenerative medicine products with plug-and-play formats for key technology areas including tissue engineering, cell therapy, bioprinting, extracellular vesicle and synthetic biology applications.

- To date, hMSCs have been used to treat over 36,000 patients globally and there are currently over 900 registered global clinical trials utilizing hMSCs, with 100 new trials added each year since 2011. The average patient requires $416 \mathrm{M}$ hMSCs during treatment.

- RoosterBio is the only company with commercially available, off-the-shelf cGMP clinical grade hMSC working cell banks, focused on shortening the product development timelines for researchers moving towards the clinic and to market.

- RoosterBio provides Process Development Services to optimize hMSC manufacturing processes, reduce cost of goods, and boost productivity for research groups developing regenerative medicine products.

- To increase its reach and impact, RoosterBio has strategically partnered with industry leaders in the cell manufacturing space to provide novel end-to-end solutions for its customers, including Gencure, ExoPharm, CELLINK, and PBS Biotech.

- In addition to industry partnerships, RoosterBio participates in several national consortia and organizations, including the National Institute for Innovation in Manufacturing Biopharmaceuticals, Standards Coordinating Body, the Alliance for Regenerative Medicine, the National Science Foundation Engineering Research Center for Cell Manufacturing Technologies, and the Advanced Regenerative Manufacturing Institute. 


\section{Financial \& competing interests disclosure}

TR Olsen and JA Rowley are employees of RoosterBio Inc., JA Rowley is the Chief Technology Officer of RoosterBio Inc. and maintains ownership. RoosterBio Inc. is a human stem cell manufacturing company focused on accelerating the cell-based bioeconomy by providing standardized stem cell product platforms that enable rapid clinical and commercial translations. This paper is the reflection of opinions generated based on industry experience and knowledge in the field. The authors have no other relevant affiliations or financial involvement with any organization or entity with a financial interest in or financial conflict with the subject matter or materials discussed in the manuscript apart from those disclosed.

No writing assistance was utilized in the production of this manuscript.

\section{References}

1. Rowley J, Abraham E, Campbell A, Brandwein H, Oh S. Meeting lot-size challenges of manufacturing adherent cells for therapy. Bioprocess Int. (2012). www.bioprocessintl.com/manuf acturing/cell-therapies/meeting-lot-size-challenges-of-manuf acturing-adherentcells-for-therapy-328093/

2. Simaria AS, Hassan S, Varadaraju $\mathrm{H}$ et al. Allogeneic cell therapy bioprocess economics and optimization: single-use cell expansion technologies. Biotechnol. Bioeng. 111(1), 69-83 (2014).

3. Olsen TR, Lock LT, Rowley JA. Scaling up: how manufacturing sciences will dictate the future of cell therapy. Regen. Med. 11(8S), S15-S18 (2016).

4. Pattasseril J, Varadaraju H, Lock L, Rowley JA. Downstream technology landscape for large-scale therapeutic cell processing. Bioprocess Int. 11, 38-47 (2013).

5. Andrew C, Thomas B, Lior R et al. Concise review: process development considerations for cell therapy. Stem Cells Transl. Med. 4(10), $1155-1163$ (2015).

6. Rowley JA, Lock LT. RoosterBio. Ready-to-print cells and integrated devices: 20170079262 (2017). https://patents.google.com/patent/US20170079262A1/en

7. Lock LT, Rowley JA. RoosterBio. Biopreserved stem cells on microcarriers: 20180216072 (2018). https://patents.google.com/patent/US20180216072A1/en

8. Lock LT, Rowley JA. RoosterBio. Containers for closed system single-use bioreactors and culture vessels; WO2016205787A1 (2016). https://patents.google.com/patent/WO2016205787A1/en?q=containers\&q=closed\&q=single\&q=use\&q=b ioreactors\&oq=containers + for + closed + single + use + bioreactors

9. NIH U.S. National Library of Medicine. ClinicalTrials.gov. www.clinicaltrials.gov

10. Cell Trials Data. www.celltrials.org

11. Olsen TR, Ng KS, Lock LT, Ahsan T, Rowley JA. Peak MSC: are we there yet? Front. Med. 5, 178 (2018). 
